\title{
Innovaciones Tecnológicas Para Inclusión Educativa De Estudiantes Sordos
}

\author{
Technological Innovations For Educational Inclusion Of Deaf Students \\ ${ }^{*}$ Clemencia Zapata ${ }^{1}$; Jairo Acosta-Solano \\ Recibido para publicación: 14 de agosto 2018 - Aceptado para publicación: 14 de octubre 2018
}

\section{RESUMEN}

En el marco de una "Educación para todos y con todos, inclusiva y diversa", se realizó el proyecto "Modelo para producción de contenidos digitales educativos para inclusión de sordos", adscrito al Programa Nacional Arquitectura Pedagógica, didáctica y tecnológica para formación de profesores en y para la diversidad, financiado por COLCIENCIAS 546-2014. El trabajo fue desarrollado en tres niveles de investigación: 1. documental y diagnóstica, 2. descriptiva, 3. de diseño y aplicada, definiendo, a su vez, seis fases metodológicas para el desarrollo e implementación del modelo para la apropiación y uso inclusivo de las TIC en todos los niveles escolares. La producción de contenidos digitales educativos se despliega a partir de la arquitectura definida para el modelo de producción, donde las comunidades de práctica interactúan en las capas estructurales de Gestión de Conocimiento, de Gestión de los Procesos pedagógico, diseño y producción, validación y apropiación y uso, y de, Gestión del Campus Virtual APROPIA-DOS. En el campus se incluyó el modelo conceptual, el formato del diseño instruccional, la normativa vigente, junto con los recursos y herramientas educativas accesibles para sordas, porque se incorporan el intérprete en lengua de señas colombianas, para garantizar aprendizajes en el marco de la inclusión educativa.

PALABRAS CLAVE: Inclusión, Educación, Diversidad, Contenidos Digitales, Didáctica.

\begin{abstract}
Within the framework of an "Education for all and with all, inclusive and diverse", the project "Model for production of educational digital contents for the inclusion of the deaf" was carried out, attached to the National Pedagogical, Didactic and Technological Architecture Program for teacher training in and for diversity, financed by COLCIENCIAS 546-2014. The work was developed in three levels of research: 1. documentary and diagnostic, 2. descriptive, 3. design and applied, defining, in turn, six methodological phases for the development and implementation of the model for the appropriation and inclusive use of ICTs at all school levels. The production of digital educational content is based on the architecture

Doctoranda en Investigación Educacional - Universidad Internacional iberoamericana, Magister en Educación - Universidad Santo Tomás, Especialista en Pedagogía para el Aprendizaje Autónomo, Licenciada en Ciencias de la Educación, Espcialidad en Química y Biología - Universidad Libre. Lider de Investigación en el área de Educación, facultad de Ciencias Sociales y Humanas en la Corporación Universitaria Rafael Núñez. clemencia.zapata@curnvirtual.edu.co.

2 Ingeniero Industrial (1997), Especialista en Finanzas (1998), Máster en Educación y TIC (2012), Maestrando en Analisis y Visualización de Datos Masivos. Líder de Investigación de la Facultad de Ingeniería en la Corporación Universitaria Rafael Núñez. Cartagena de Indias (Colombia)
\end{abstract}


defined for the production model, where the communities of practice interact in the structural layers of Knowledge Management, Management of Pedagogical Processes, Design and Production, Validation and Appropriation and Use, and Management of the APROPIA-DOS Virtual Campus. The campus included the conceptual model, the format of the instructional design, the regulations in force, together with the resources and educational tools accessible to the deaf, because the interpreter in Colombian sign language is incorporated to guarantee learning within the framework of educational inclusion.

KEY WORDS: Inclusion, Education, Diversity, Digital Content, Didactics.

\section{INTRODUCCIÓN}

Esta investigación coadyuva con la Política Nacional propuesta en "Paz, equidad y Educación" acogida por Ciencia, Tecnología e Innovación, una sinergia lograda entre Pedagogía e Ingeniería, sustentada la gestión del proyecto en el trabajo integral desarrollado por las comunidades de práctica pedagógica, tecnológica, didáctica y de gestión de conocimiento (Wenger y Barberán, 2001).

Se acoge la diversidad como una dinámica social que articula procesos de reconocimiento de promoción, cooperación y crecimiento con lo otro, con los otros y con sus diferencias, desde esta perspectiva, el sistema educativo reconoce el derecho a la igualdad [2], al acceso de formas de explorar, construir y comunicar conocimiento con todos y para todos; valorando la diversidad como una riqueza propia de la humanidad.

Bajo esa intencionalidad, se propuso, "Desarrollar modelos para la apropiación y el uso de tecnologías en escenarios educativos que acogen diversidad de poblaciones", atendiendo la siguiente ruta: 1. Identificar y determinar el enfoque epistemológico, teórico y metodológico del modelo a partir de una línea base que caracterice al educando sordo, los estándares curriculares, los estilos y objetivos de aprendizaje; 2. Diseñar un modelo instruccional para la apropiación y uso de TIC que permita potenciar los procesos pedagógicos que medien la inclusión de sordos en contextos educativos. 3. Implementar el modelo desplegando la producción y usabilidad de recursos digitales para sordos como apoyo a las mediaciones didácticas propuestas y desarrolladas por los docentes; y, 4. Valorar el impacto pedagógico y didáctico del modelo y su potencial de apropiación y uso en las escuelas con sordos.

Con estas premisas, la metodología desplegada para la realización de este proyecto, da cuenta de una Investigación aplicada, que incluye una investigación documental y diagnóstica, desplegada en fases: Fase I. Encuadre Epistemológico, teórico y metodológico; Fase II. Caracterización de aprendizajes; Fase III. Diseño del modelo con estrategias Pedagógicas y Tecnológicas; Fase IV. Desarrollo e Implementación del modelo; Fase V. Valoración de impacto; Fase VI. Divulgación.

Se inicia la consolidación de la Línea base por los grupos de investigación Sistemas Neurodifusos y Huellas Pedagógicas de la Corporación Universitaria Rafael Núñez, caracterizando las instituciones educativas, docentes y población sorda, para identificar: (1) La infraestructura tecnológica de las instituciones y el nivel de conocimiento en TIC de docentes de sordos; (2) Los estándares y objetivos de aprendizaje para la población sorda; (3) Las teorías pedagógicas y metodológicas vigentes en contextos inclusivos; (4) Los modelos pedagógicos de las instituciones educativas que atienden población sorda; (5) Los estilos de aprendizaje de la población sorda; (6) Aspectos administrativos, recursos educativos y políticas existentes en las escuelas que atienden sordos.

Lo anterior, develó el bajo nivel de apropiación de los conceptos de entornos y objetos virtuales de aprendizaje, tanto en profesores como en estudiantes, limitaciones en cuanto al uso de recursos didácticos que motiven y potencien el aprendizaje de sordos en estos contextos educativos inclusivos; deficiencias en infraestructura y recursos tecnológicos; heterogeneidad marcada en cuanto a diagnóstico y edad de los estudiantes sordos; insuficiencia en cuanto a adecuaciones concretas en los planes de estudio para sordos; pocas investigaciones, en particular ninguna sobre estilos cognitivos de la población en condición de discapacidad auditiva.

\section{MÉTODOS}

Acoger la diversidad se entiende como la dinámica social que articula procesos de reconocimiento 
de promoción, cooperación y crecimiento con lo otro, con los otros y con sus diferencias, desde esta perspectiva, el sistema educativo reconoce el derecho a la igualdad [2], al acceso de formas de explorar, construir y comunicar conocimiento con todos y para todos; valorando la diversidad como una riqueza propia de la humanidad.

En este sentido en Colombia, ha sido necesario repensar la práctica docente, por los nuevos desafíos pedagógicos, didácticos y tecnológicos, implicados en las formas de operacionalizarla en la escuela, teniendo en cuenta el paradigma de complejidad, trabajar con y para diversidad, en el marco de la inclusión [3], más, de la sostenibilidad y mejora de la calidad educative [4].

Desde esas perspectivas, es desafiante el proceso de enseñanza, urge la necesidad de desarrollar capacidades y competencias en el profesorado, para atender la singularidad, las necesidades individuales de niños, jóvenes, adultos, campesinos, grupos étnicos, personas con limitaciones físicas, sensoriales, cognitivas y psíquicas, más, aquellos que requieran rehabilitación e inclusión social y educativa.

Estas lecturas, integraron la "Alianza de Instituciones para el Desarrollo de la Educación y la Tecnología en Colombia" AIDETC, para desarrollar en cuatro unidades de investigación (fig.1) un programa nacional financiado por Colciencias, en contribución a la formación de profesores:

Unidad 1. Una didáctica para la formación de profesores de matemática en contextos de educación inicial - Universidad del Quindío.

Unidad 2. Estado del arte a nivel nacional de la formación de pedagogía infantil en y para la diversidad - Corporación Universitaria Iberoamericana.

Unidad 3. Modelo para la producción de contenido digital educativo para la inclusión de sordos Corporación Universitaria Rafael Núñez.

Unidad 4. Desarrollo didáctico y tecnológico en escenarios didácticos para la formación Universidad Distrital Francisco José de Caldas.

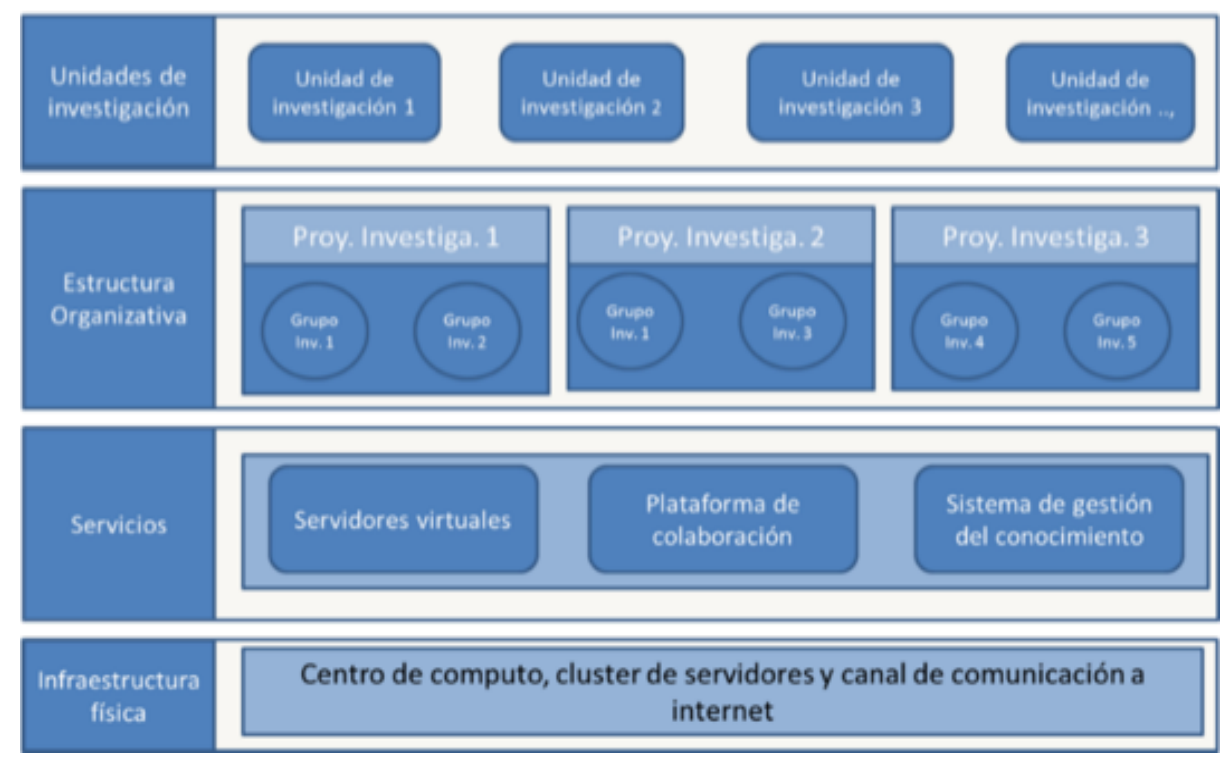

Figura 1. Arquitectura organizacional y tecnológica del proyecto. Fuente propia

El programa desarrolló una investigación aplicada, soportada en las fases mostradas en la Figura 1, para dar cuenta del objetivo principal "Constituir una infraestructura pedagógica, didáctica y tecnológica para la creación y el desarrollo de escenarios educativos, a partir de la investigación sobre ambientes de enseñanza y aprendizaje de estudiantes para acoger y promover la educación de poblaciones marginadas y en condición de vulnerabilidad", logrado con:

1. El diseño e implementación de un modelo para la apropiación y el uso de tecnologías en escenarios educativos que acogen diversidad de poblaciones.

2. La consolidación de una infraestructura pedagógica y didáctica en escenarios tecnológicos para diseñar, Implementar y validar ambientes didácticos de aprendizaje

3. Caracterización de los procesos de gestión de conocimiento (GC) al interior de las comunidades de práctica.

\subsection{Organización y Formación.}

Las actividades se organizaron en un plan 
operativo, para controlar y evidenciar los avances de la investigación, en el se articuló dento del sistema de gestión de conocimiento del programa, la estructura y el desarrollo del plan de capacitación a los investigadores en manejo de infraestructura tecnológica de soporte, coa cargo de la Universidad Distrital Francisco José de Caldas, se incluyó: software Microsoft Project, uso de Plataformas Virtuales de Aprendizaje A-Tutor, Liferay Plataforma de Colaboración; con esa capacitación se garantizó el diseño, desarrollo y ejecución de los cursos virtuales, para la formación de profesores; la producción de recursos educativos digitales. http://aidetc.redalternativa.org/ .

\subsection{Desarrollo e implementación de modelos} (Fig. 2).

Este objetivo se logró con el desarrollo del proyecto "Modelo de Producción de contenidos digitales para sordos", a cargo de los grupos de investigación Huellas Pedagógicas y Sistemas Neurodifusos de la Corporación Universitaria Rafael Núñez. Se fundamentó en los resultados arrojados por la Línea Base descrita en la introducción. Esas razones, justifican en más, la necesidad de diseño conceptual del Modelo (fig. 2), además de permitir al equipo de investigadores refinar una innovación en el diseño instruccional propuesto por Merrill [5]; se logró consolidar un curso para producción de contenidos digitales educativos, específicamente construcción de Objetos Virtuales de Aprendizaje (OVA), y una rúbrica de evaluación para REDA. Con esa aproximación, se inició la producción de recursos digitales, para formación de docentes y para mejorar procesos de aprendizaje de la población sorda.

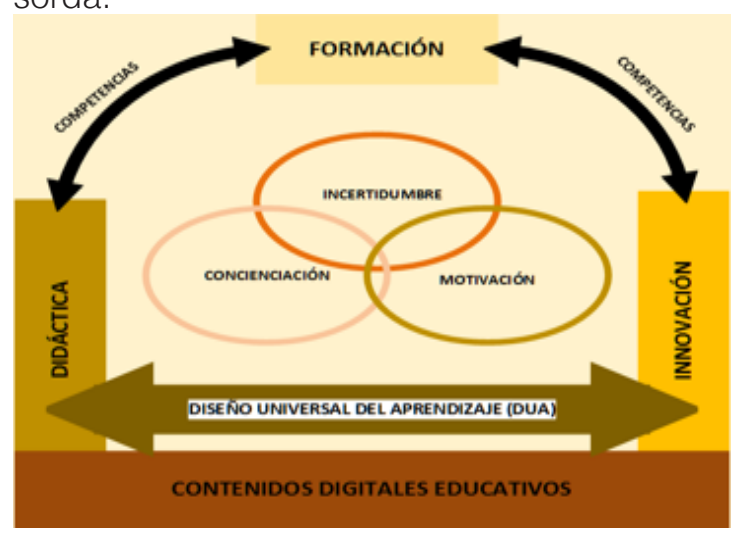

Fig. 2. Diagrama Modelo para producción de contenidos digitales educativos. Fuente propia.
http://apropiados.curnvirtual.edu.co/\#!/Conceptualizacion

\subsection{El carácter del modelo:}

Se asume desde la perspectiva formativa, porque la prevalencia del gran propósito del programa de formar profesores para mejorar su desempeño laboral en escenarios de enseñanza y de aprendizaje, formándolos en competencias TIC, desde la perspectiva del MEN. "El futuro de la formación con soporte tecnológico" [6]; el modelo es didáctico porque se considera un instrumento de análisis, intervención y mejora de la práctica educativa, basado en el desarrollo de metodologías de "investigación escolar", de trabajo en torno a "problemas", con secuencia de actividades relativas al tratamiento de esos problemas, donde el profesor es coordinador de los procesos porque funge como "investigador en el aula" [7]; y, es Innovador, porque propende por una "cultura innovadora" donde los docentes innovadores se auto-reconocen como tales, están abiertos a transformar su práctica, se arriesgan, planifican y son novedosos en sus estrategias de cambio y, trabajan en equipo hacia una meta común [8].

El modelo está fundamentado en el principio de Incertidumbre [9] por los vertiginosos cambios del mundo que impactan en los sistemas educativos, este hecho, avoca a las comunidades a mejorar la calidad de la educación en el marco de la libertad pedagógica, bajo la promesa de formar educadores de excelencia, y además, de inducir una enseñanza reflexiva en el amrco de la incertidumbre. De otro lado, con Edgar Morin [10] "Hay que aprender a enfrentar la incertidumbre puesto que vivimos una época cambiante donde los valores son ambivalentes, donde todo está ligado. De ahí que la educación del futuro debe trabajar las incertidumbres ligadas al conocimiento", ello avoca a proponer cambios sustanciales en el conocimiento de las ciencias, la educación, la cultura y la sociedad.

Se consideró determinante incluir la teoría de la decisión [11], decisión con incertidumbre o riesgo, una teoría de juegos, que bien se enmarca en las posibilidades del contexto educativo y de los sujetos que en ellos interactúan. Desde otros ángulos, el modelo pone en juego el fenómeno de la concienciación [12], relacionado con la conciencia fenoménica y las posturas ficisistas y antifisicistas que se revelan en los contextos formativos; en complemento se incluyen planteamientos teóricos sobre la motivación en el proceso de enseñanza y de aprendizaje, la 
búsqueda de intereses bajo las opciones de mantenimiento del esfuerzo, de persistencia, y de autorregulación.

Se incorporan en el modelo, los principios y objetivos del Diseño Universal de Aprendizaje [13], en un intento por visibilizar la inclusión de lo diverso y de validar la necesidad de generar recursos y ambientes educativos abiertos y accesibles; también es obligado integrar el sistema de competencias TIC recomendadas por el MEN para el desarrollo profesional docente: Tecnológica, Pedagógica, Comunicativa, de Gestión e Investigativa [14].

\subsection{Roles y responsabilidades.}

Se establecieron niveles de responsabilidad (Fig. 3), para garantizar de la obtención de los objetivos propuestos.

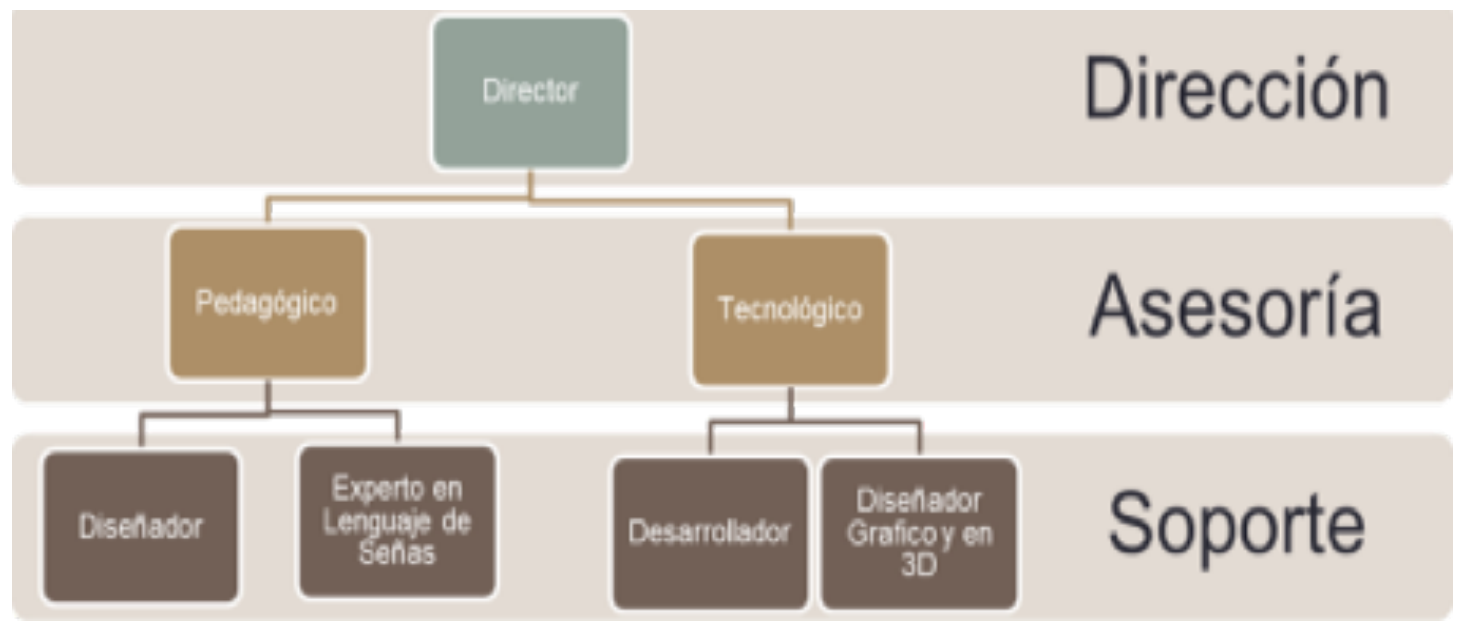

Figura 3. Roles y responsabilidades del equipo de trabajo. Fuente propia

El modelo para la producción de contenido digital educativo se desplegó en cuatro componentes
(Fig. 4), que representan los subsistemas del sistema global, los mecanismos de integración, las posibles interacciones y dependencias entre ellos

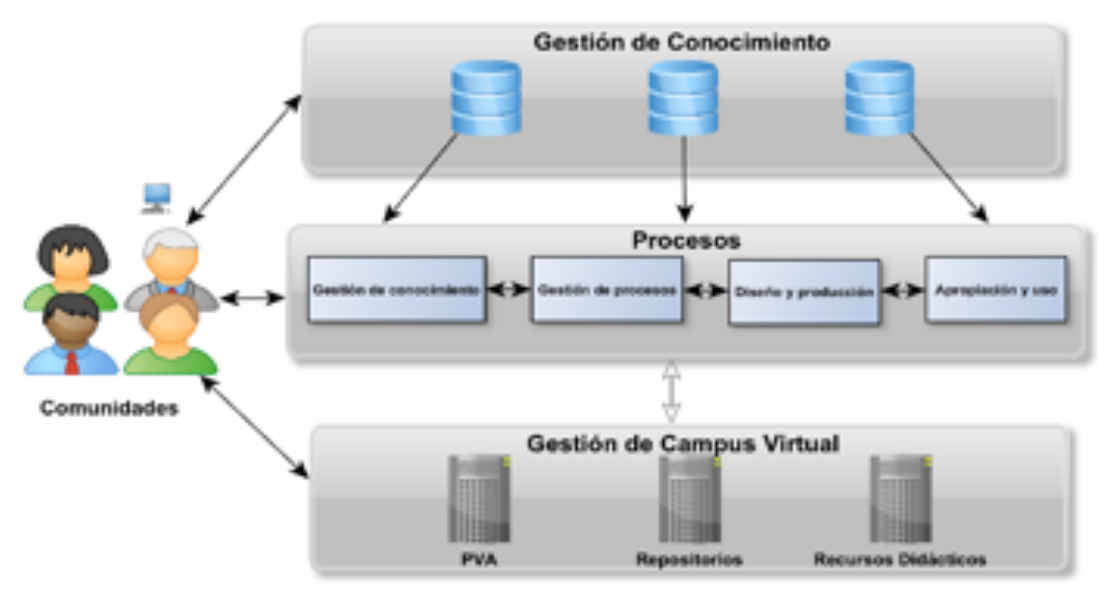

Figura 4 Componentes del Modelo de producción de contenidos digitales educativos Fuente: propia. 
y las tecnologías requeridas para su soporte.

\section{COMUNIDADES DE PRÁCTICA [1]}

Los docentes y directivos en sus prácticas tienen la responsabilidad de incluir y asumir a los estudiantes en condición diversa, como oportunidad para dignificarlos en marco de una cultura para y por la paz, en virtud del principio de equidad educativa, de una educación para todos y de calidad.

Se generaron comunidades didácticas, tecnológicas y de gestión de conocimiento y otras disciplinares; y, una comunidad de sordos: profesores sordos, sordos autónomos, y, otras comunidades educativas.

\section{GESTIÓN DE CONOCIMIENTO}

Realimenta el conocimiento de las comunidades de práctica [15], visibiliza los intereses particulares de conocimiento de estas, su condición, su nivel de uso de las TIC, su evolución y su motivación hacia el uso de otros componentes del modelo en un proceso de retroalimentación del mismo.

Investigaciones realizadas en países angloparlantes encuentran importante aprovechar las TIC en el aprendizaje del estudiante, en la mejora de las competencias TIC del professor, en sus creencias, expectativas, flexibilidad al cambio y prácticas pedagógicas; se infiere que estos factores tienen el mismo peso en Iberoamérica. La gestión de conocimiento integra en una base de datos, la identificación, el análisis de requerimientos, de contenidos y productos, la validación de productos y verifica la apropiación TIC en las comunidades de práctica.

\section{GESTIÓN DE PROCESOS}

Este componente mantiene la lógica desarrollada en los procesos didácticos y pedagógicos, curriculares, de diseño y de producción, validación de contenidos, de uso y de apropiación de TIC por las comunidades de aprendizaje. Los procesos allí, se documentan y señalan los participantes, las actividades realizadas, la toma de decisiones, y cuentan con soporte documental normado, instructivos y procedimientos.

\section{PROCESO DISEÑO $Y$ PRODUCCIÓN DE CONTENIDOS (FIG. 5)}

Los docentes apropiados en manejo de las TIC que son soporte educativo, deben capacitarse en el diseño universal de aprendizaje, para producir recursos didácticos: Objetos Virtuales de Aprendizaje, Objetos Virtuales de Información, repositorios, Web 2.0, videojuegos, libros digitales. Las sensores espaciales son la fuente de monitoreo a gran escala en tiempos regulares. De ellos se desprenden diferentes métodos que cuantifican el cambio dentro de estos se encuentran los índices de vegetación NDVI, variables climatológicas, los modelos lineales generalizados (GLM) y los estadísticos de confiabilidad y variabilidad espectral (divergencia transformada, gráficas de signaturas y matrices de exactitud temática).

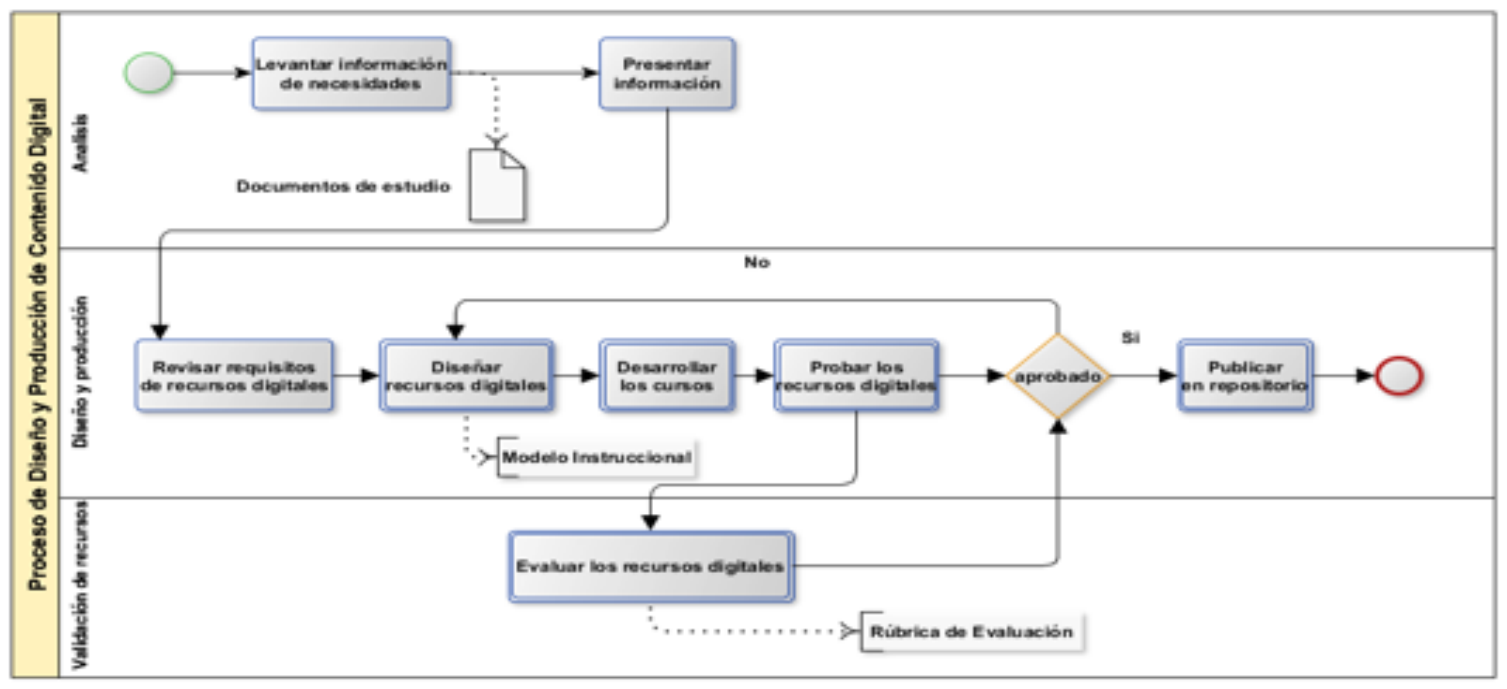

Figura 5 Proceso diseño y producción de contenido digital. Fuente propia. 


\section{PROCESO DE APROPIACIÓN Y USABILIDAD DE LAS TIC}

El docente en su desarrollo profesional debe integrar las TIC en el proceso de enseñanza/ aprendizaje, demostrando: a) competencia básica en uso de las TIC, b) disposición de estrategias pedagógicas para utilizar el potencial transformador de las TIC en la mejora de los aprendizajes, c) integrar efectivamente las TIC en su disciplina, d) Generar competencias investigativas, para innovar en el aula de clases, e) gestionar la consecución de recursos TIC; las anteriores competencias permiten reconocer su nivel en el uso pedagógico de las TIC: 1. Explorador, 2. Integrador, 3. Innovador (fig. 6).

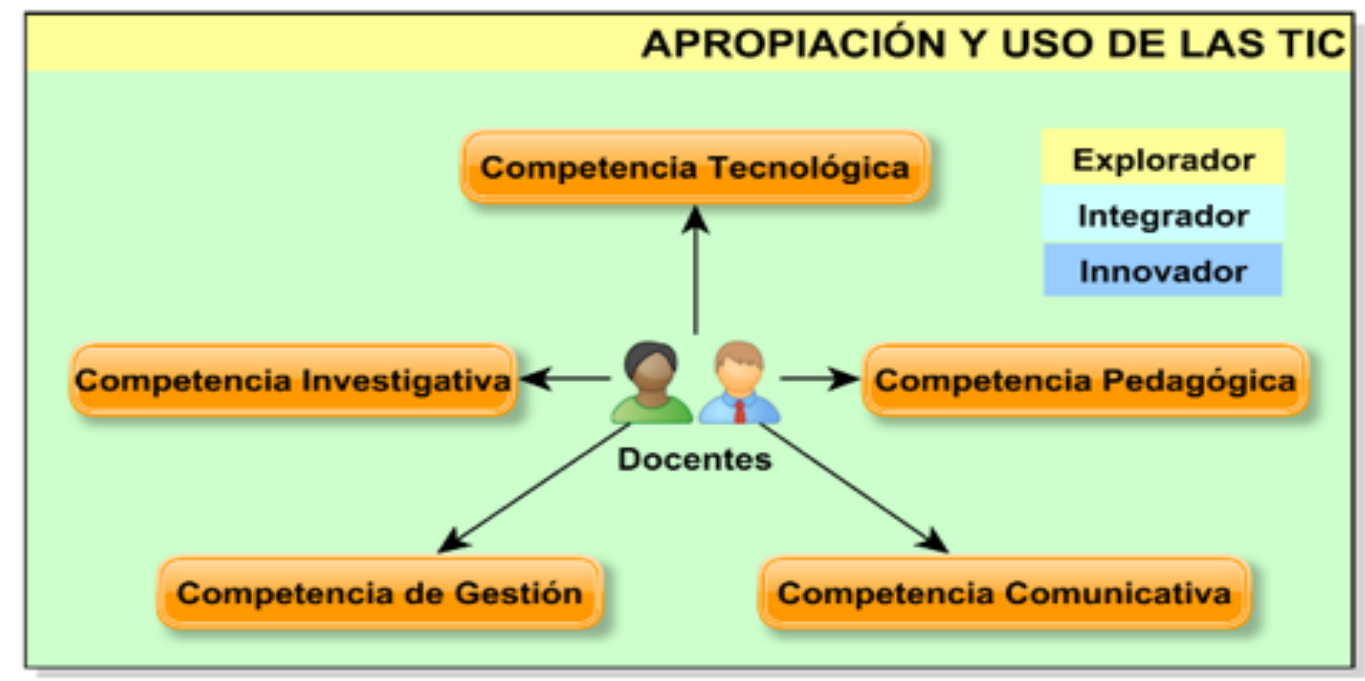

Figura 6. Competencias del desarrollo profesional docente. Fuente propia.

\section{GESTIÓN DEL CAMPUS VIRTUAL}

http://apropiados.curnvirtual.edu.co/\#!/

El campus virtual es el componente nuclear que provee la interfaz web para acceso de las comunidades a las plataformas virtuales de aprendizaje; se constituye en medio de acceso a los recursos didácticos en repositorios de objetos virtuales de aprendizaje, de vídeo juegos y libros digitales, para potenciar la enseñanza y el aprendizaje en los sordos y oyentes.

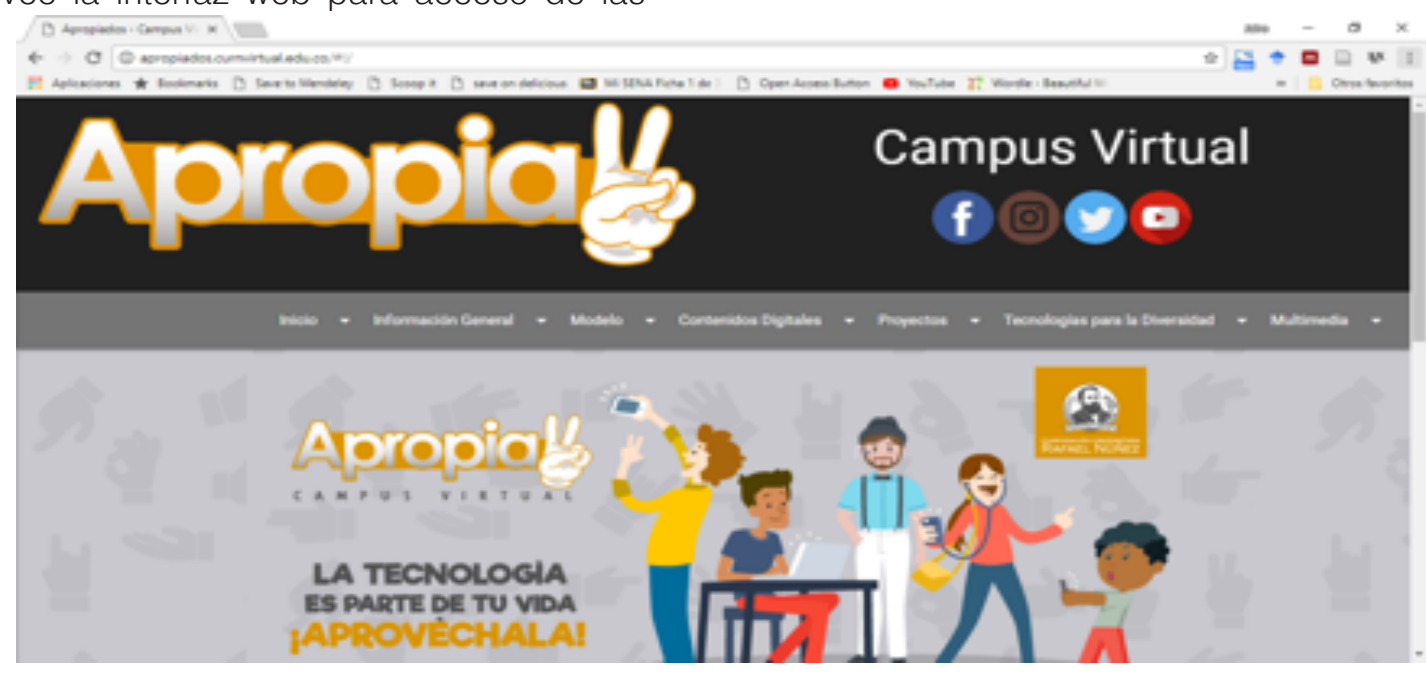

Figura 7 Interface campus virtual. Fuente propia 
El campus virtual cuenta con plataforma para cursos, repositorios de recursos educativos digitales (fig. 8), dispone de un intérprete de lengua de señas colombiana (LSC), de un prototipo de avatar "HADO" que se comunica en LSC, sistema closed caption, subtitulación, modelos lingüísticos y cuenta con dispositivos para mejorar la accesibilidad visual.

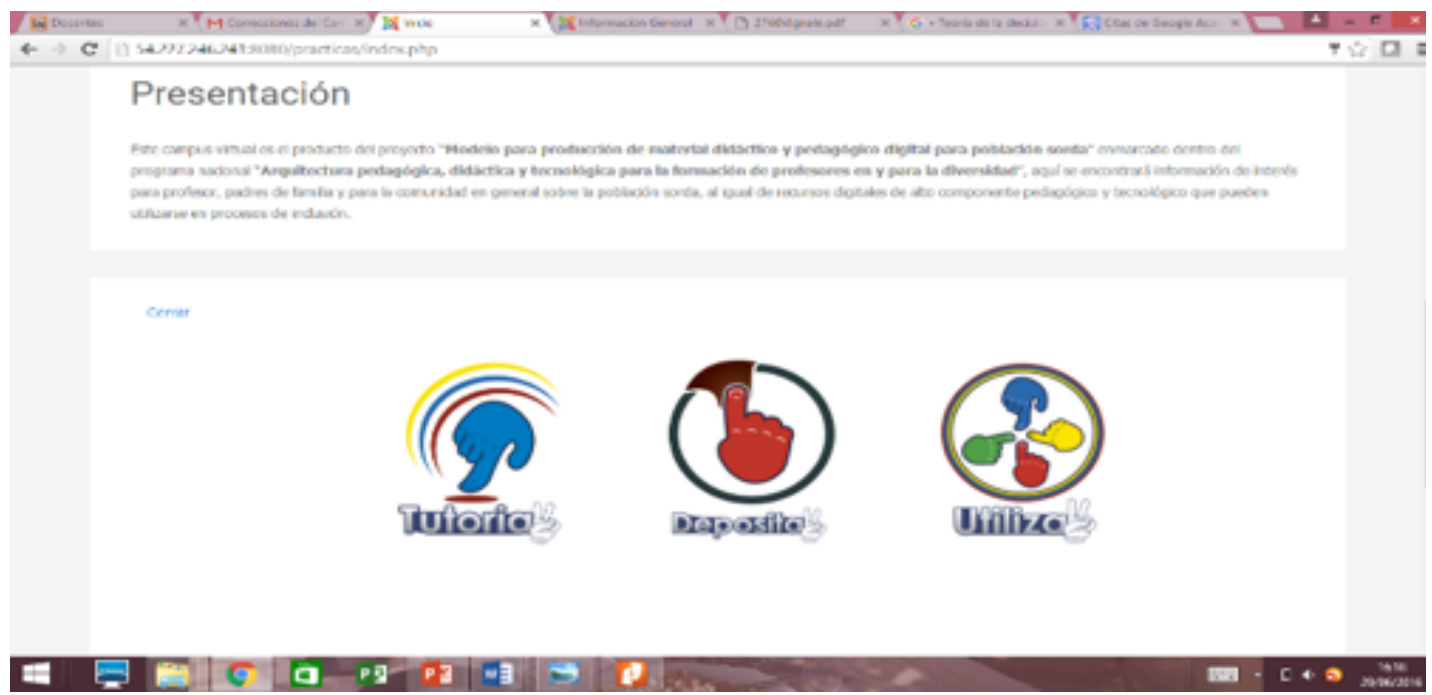

Figura 8. Plataformas con recursos digitales educativos. Fuente propia.

En la plataforma tutoria-dos (Fig 8.) se incorporan de aprendizaje, accedan y aprendan a diseñar cursos: http://apropiados.app.curnvirtual.edu. co/cursos, allí los miembros de comunidades y desarrollar diversos contenidos y recursos digitales de aprendizaje (fig. 9).

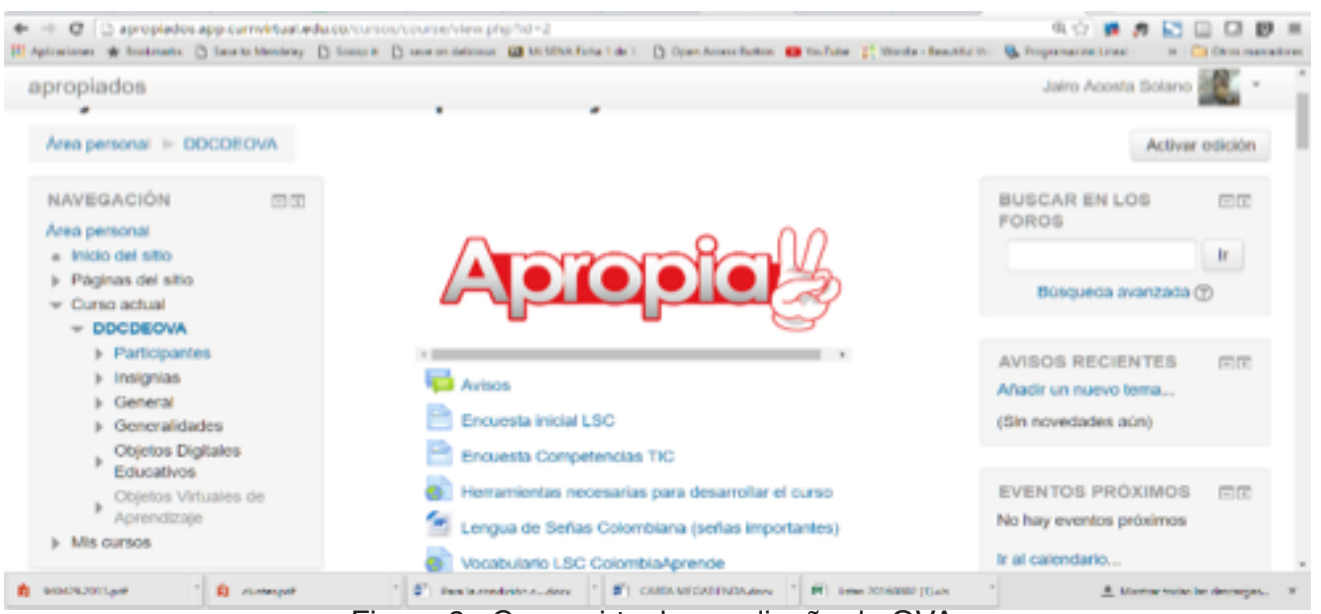

Figura 8. Curso virtual para diseño de OVA.

El diseño instruccional (Fig.10) es un esquema sistémico, planificado y estructurado, que permite producir contenidos digitales para educación virtual o presencial; a nivel formativo, se puede presentar en módulos o unidades didácticas para recrear escenarios de aprendizaje [15]. http://apropiados.curnvirtual.edu.co/\#!/ Dise\%C3\%B10\%20Instruccional\%20Cognitivo 


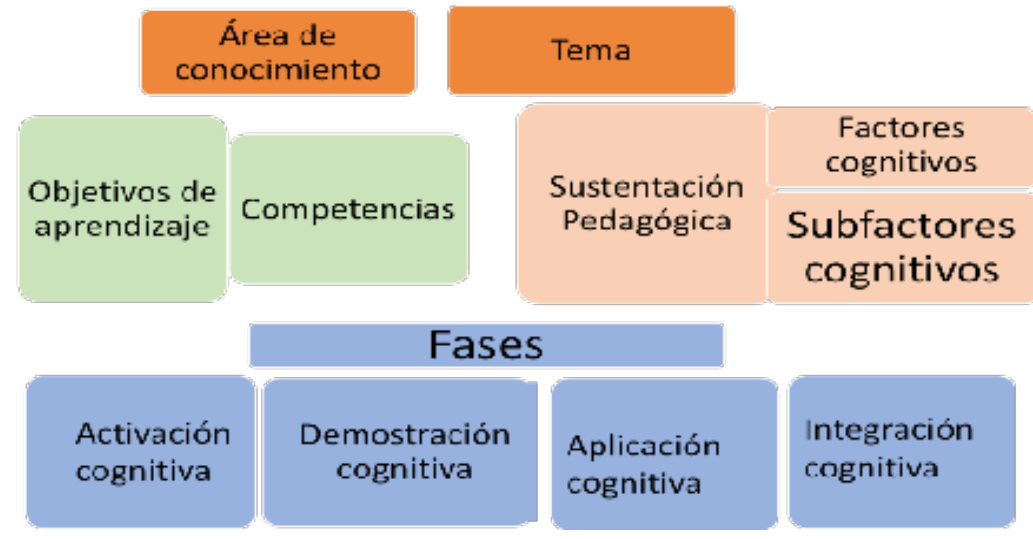

Figura 10. Plantilla del diseño Instruccional Cognitivo. Fuente propia

El diseño instruccional permite al professor preparar su clase, organizar la secuencia didáctica, las actividades y los contenidos en perfecta correlación, reconocer la naturaleza de la tarea y garantizar que sus estudiantes identifiquen, entiendan y comprendan el proceso mental implícito en la enseñanza y el aprendizaje tanto en sordos como en oyentes.

En el Proyecto Modelo para producción de Contenidos Digitales Educativos, se produjeron cinco (5) Objetos Virtuales de Aprendizaje en versión HTML; se usó computación inmersiva e inteligencia artificial.

La fig. 11 enseña la técnica de reanimación cardiopulmonar, incluye la intérprete en lengua señas colombiana, para apoyar el proceso de aprendizaje a tecnólogos en Atención Prehospitalaria, enfermeros, médicos y personas en condición de discapacidad auditiva. http:// apropiados.curnvirtual.edu.co/Productos $\% 20$ Finales/Produccion\%20tecnologica/OVA/RCP/

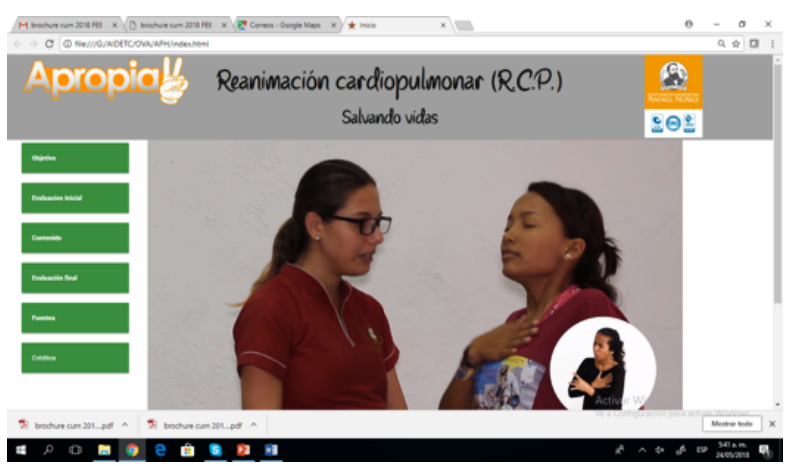

Figura 11. OVA Reanimación Cardiopulmonar Salvando vidas Fuente Propia

EI OVA de la Fig. 12 se realizó junto con la compañia de baile BONDI en Buenos Aires-Argentina para enseñar a bailar a personas sordas, integra la secuencia de vídeos con el proceso completo.
Este recurso educativo virtual puede ser usado en escuelas y facultades de arte. http://apropiados. curnvirtual.edu.co/Productos\%20Finales/ Produccion\%20tecnologica/OVA/Bondi/

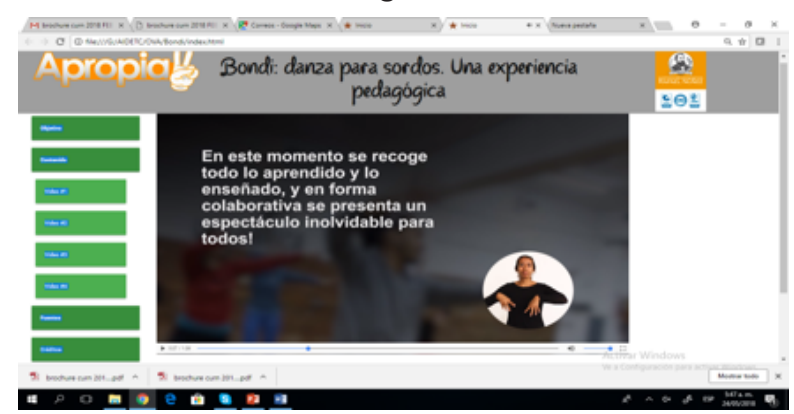

Figura 12. OVA Bondi: Danza para sordos. Fuente propia.

El OVA Cartagena histórica y virtual (Fig.13) incluye monumentos históricos de Cartagena de Indias, para usar en clases de sociales, cátedra de historia a nivel de primaria y puede ser un modelo para producir otros contenidos por estudiantes de Programas de Educación para mejorar y formentar sus competencias digitales. http://apropiados. curnvirtual.edu.co/Productos $\% 20$ Finales/ Produccion\%20tecnologica/OVA/OVA\%20-\%20 Sociales/

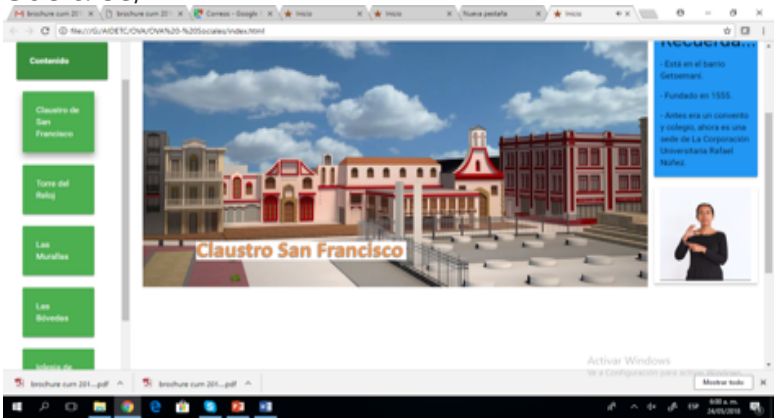
Figura 13. OVA Cartagena virtual e histórica. Fuente propia.

El OVA Kick Ball, si al deporte (Fig 14) se soporta en la técnica "Draw my class"; enseña a niños 
y estudiantes las bases del juego regional, se propone para la clase de educación física. La intención es quebrar los imaginarios en torno a su construcción. http://apropiados.curnvirtual. edu.co/Productos\%20Finales/Produccion\%20 tecnologica/OVA/OVA\%20KickBall/

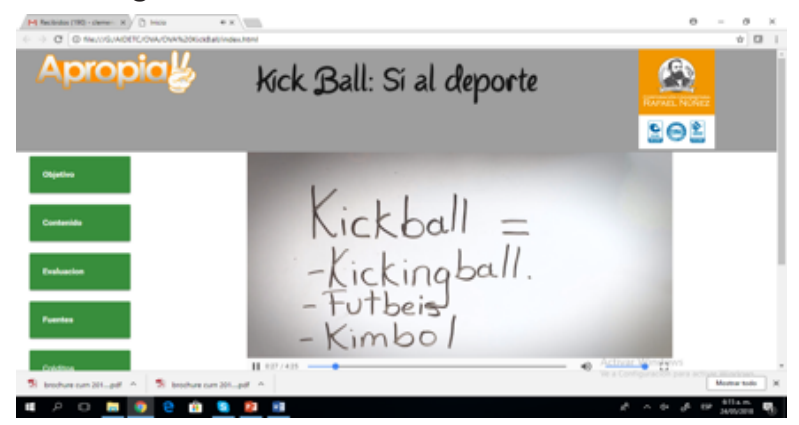

Figura 14. OVA Kick Ball: Si al deporte. Fuente propia.

EL OVA de matemáticas (fig 15), enseña a contar en prematemáticas $01^{\circ}$ primaria, su diseño instruccional, da gran importancia al conteo, por su influencia en el desarrollo de la capacidad para tomar decisiones. http://apropiados.curnvirtual. edu.co/Productos\%20Finales/Produccion \%20 tecnologica/OVA/OVA_MATH/

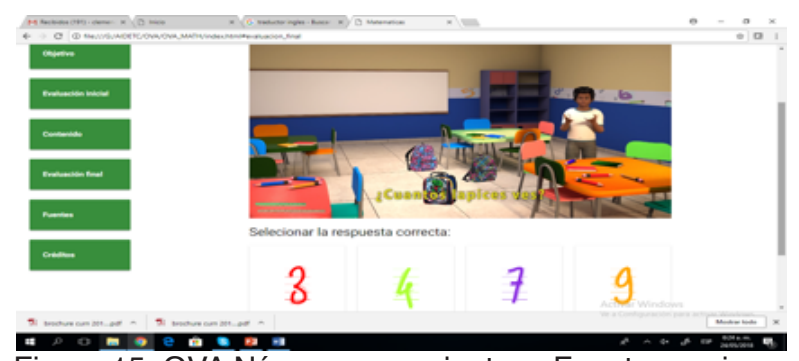

Figura 15. OVA Números y conjuntos. Fuente propia.

Se desarrollaron dos vídeojuegos en motor de vídeo juego Unity 3D, diseñados y construidos conceptulamente desde el juego y la necesidad del estudiante sordo, caracterizada por modos de funcionamiento socio-culturales y cognoscitivos diferentes a los oyentes [19], reglas que gobiernan el contacto y los roces físicos; uso de expresiones faciales y gesticulación y convenciones derivadas de la significación de la visión y del entorno. Es una actitud, una experiencia de vida diferente [16].

El equipo de investigadores consideró los videojuegos y sus dispositivos específicos, útiles para mejorar el proceso de aprendizaje del sordo, son atractivos; aumentan sin presión, ni estrés: el desarrollo de habilidades cognitivas, dedican más tiempo al autoaprendizaje, la motivación hacia el aprendizaje, los niveles concentración y de atención mientras trabajan y jugando [17]. Se destaca, la importancia de incorporar en los programas educativos herramientas históricotecnológico contemporáneas, para enfrentar el futuro con las competencias necesarias [18].

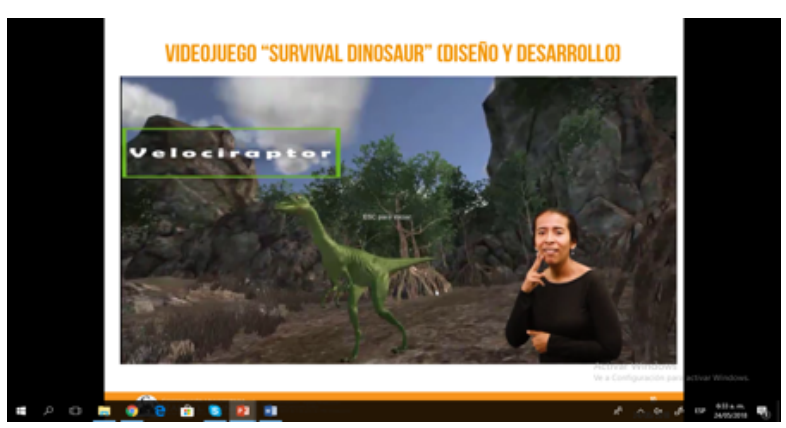

Figura 16. Vídeo-juego la vida de los dinosaurios Fuente propia.

La vida de los dinosaurios (fig. 16) es recreada en secuencias didácticas [20], para el aprendizaje de un tema en ciencias naturales; el segundo aborda las señales de tránsito (fig. 17) desde la pedagogía social [20].

Estos contenidos digitales son una respuesta a las necesidades identificadas por el equipo de investigadores, en la línea base ques e levanto e incluyó, el estudió de los estilos de aprendizaje, los estándares curriculares de finidos para la población sorda, estos recursos permiten superar las barreras de aprendizaje develadas.

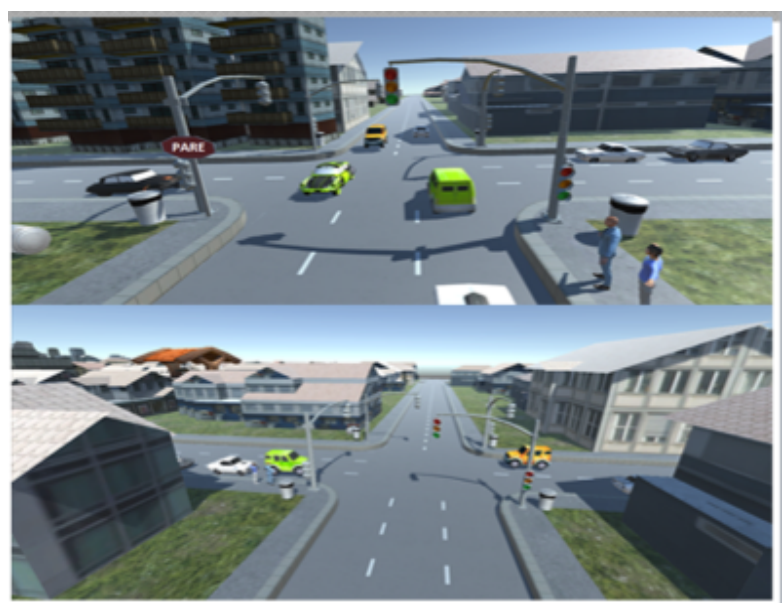

Figura 17. Videojuego Las señales de tránsito Fuente propia.

\subsection{Validación y difusión}

Todos los recursos desplegados en los contenidos digitales educativos, tuvieron validación por pares expertos, y, validación social en el campus virtual Apropiados (Fig. 7) en una fase de pruebas; 
además, en el campus se integró un instrumento para identificar el interés de los usuarios, la conformación de comunidades de práctica [1] y otro, para validación de la usabilidad de Iso recursos.

\section{CONCLUSIONES}

- El proyecto generó impacto nacional, con la integración de cuatro instituciones de educación superior de Zona Cafetera, Zona Caribe y en la Región Andina para promover una educación para todos, equidatativa y para la paz en Colombia, con el desarrollo del programa Nacional Arquitectura pedagógica, didáctica y tecnológica para formación de profesores en y para la diversidad, consolidando la red académica AIDETC.

- Se fortalecieron las líneas de investigación de los grupos de investigación, las comunidades educativas y las instituciones que incluyen estudiantado con sordera.

- Contribuyó a la formación de docentes de estudiantes sordos, con un curso para producir contenidos educativos digitales inclusivos, fortaleciendo las capacidades y de habilidades intelectuales y tecnológicas de los maestros, como aporte a la necesidad de formación en TIC.

- Los profesores de estudiantes sordos reconocieron el potencial didáctico de los contenidos digitales educativos producidos, los valoran por el aumento de la motivación, del interés y del grado de atención y concentración alcanzado.

- El Instituto Nacional de Sordos se vinculó como entidad de apoyo, acompañó la fase de pilotaje de los cursos para diseño y producción de contenidos digitales educativos para sordos y en las pruebas aplicadas sobre estilos cognitvos y de aprendizaje.

- El campus virtual es gestor de contenidos, concentrador de actividades para la mejora de las competencias profesionales del docente; es un proveedor de recursos didácticos digitales para estudiantes sordos y regulares; se potencia como generador de comunidades de aprendizaje y, modelador de relaciones entre sus usuarios.

- El trabajo interdisciplinario integró los campos teóricos y procedimentales de la pedagogía y la ingeniería.
- El estudio posibilitó conocer la fundamentación política, filosófica, pedagógica y didáctica para integrarla con la tecnología en la producción del contenido digital educativo.

- Se propició la gestión de conocimiento y de proceso en el proyecto con la creación de comunidades de aprendizaje.

- Se integraron los lineamientos curriculares del Ministerio Nacional de Educación en la producción de contenidos digitales educativos con el modelo producción y las competencias docentes en TIC.

- Faltó profundizar la integración del principio de la incertidumbre y los del Diseño Universal de Aprendizaje.

- Con la integración de tecnología 3D y efectos audiovisuales es posible captar la atención del jugador, estimular la atención, potenciaro el estilo de aprendizaje visual y kinésico del sordo.

- Los videojuegos favorecen el aprendizaje, aumentan habilidades cognitivas, el tiempo dedicado a aprender, la motivación para aprender, su concentración y atención.

- Se recomienda a los profesores integrar en los contenidos digitales educativos, los objetivos y el contenido disciplinar determinado por el plan de estudios.

- El modelo queda como: referente para formación de profesores, opción para producir recursos didácticos inclusivos, basados en pedagogía interactiva, lúdica, innovadora e incluyente.

\section{REFERENCIAS}

[1]. Armenteras, D., Retana, J., Molowny, R., Roman, R., Gonzalez, F., y Morales, M. 2011a. Characterizing fire spatial pattern interactions with climate and vegetation in Colombia. Agricultural and Forest Meteorology. 151(3):279-289.

[2]. Armenteras, D., Rodríguez, N., Retana, J., y Morales, M. 2011b. Understanding deforestation in montane and lowland forests of the Colombian Andes. Environmental Change. 11(3):693-705.

[3]. Armenteras, D., Cabrera, E., Rodríguez, N., y Retana, J. 2013. National and regional 
determinants of tropical deforestation in Colombia. Environmental Change. 13(6):1181-1193.

[4]. Bodart, C., Hugh, E., Beuchle, R., Rastislav, R., Simonetti, D., Stibig, H., Brink, A., Lindquist, E. y Achard, F. 2011. Preprocessing of a sample of multi-scene and multi-date Landsat imagery used to monitor forest cover changes over tropics. Journal of Photogrammetry and Remote Sensing. 66(5):555-563.

[5]. Brovkin, V., Sitch, S., Von-Bloh, W., Claussen, M., Bauer, E. y Cramer, W. 2004. Role of Land Cover change for atmospheric CO2 increase and climate change during the last 150 years. Global Change Biology. 10(8):1253-1266.

[6]. Evangelista V., López, J., Caballero, J. y Martínez, M. 2010. Patrones espaciales de cambio de cobertura y uso del suelo en el área cafetalera de la Sierra Norte de Puebla. Investigaciones Geográficas, Boletín del Instituto de Geografía. 72:23-38.

[7]. Friedl, M., Brodley, C. y Strahler, A. 2002. Maximizing Land Cover classification accuracies produced by decision trees at continental to global scales. IEEE Transactions on Geoscience and Remote Sensing. 37(2):969-977.

[8]. Hantson, S., Chuvieco, E., Pons, X., Domingo, C., Cea, C., More, G., Cristobal, J., Peces, J. y Tejeiro, J. 2011. Cadena de pre-procesamiento estándar para las imágenes Landsat del Plan Nacional de Teledetección. Revista de Teledetección. 36:51-61.

[9]. Mas, J., Velázquez, A., Díaz, J., Mayorga, R., Alcántara, C., Bocco, G., Castro, R., Fernández, T. y Pérez, A. 2004. Assessing land use/cover changes. A nationwide multidate spatial database for Mexico. International Journal of Applied Earth Observation and Geoinformation. 5(4):249-261.

[10]. Medina, W., Macana-García, D. y Sánchez, F. 2015. Aves y mamíferos de bosque altoandino-páramo en el páramo de Rabanal (Boyacá-Colombia). Ciencia en
Desarrollo. 6(2):185-198.

[11]. Molina, G. y Albarran, A. 2013. Análisis multitemporal y de la estructura horizontal de la cobertura de la Tierra: Parque Nacional Yacambú, estado Lara, Venezuela. Cuadernos de Geografía - Revista Colombiana de Geografía. 22(1):25-40.

[12]. Moncada-Rasmussen, D. 2010. Análisis espacio-temporal del cambio en los bosques de roble (Quercus humboldtii Bonpl.) y su relación con la alfarería en Aguabuena (Ráquirá-Boyacá). Colombia Forestal. 13(2): 275-298.

[13]. Palacios-Morera, M. 1992. Cambio ambiental, teledetección y sistemas de información geográfica temporal. Actas del $\vee$ Coloquio de Geografía Cuantitativa. Universidad de Zaragoza, España. p291304.

[14]. Pérez, M. y García, M. 2013. Aplicaciones de la teledetección en degradación de suelos. Boletín de la Asociación de Geógrafos Españoles. 61:285-308.

[15]. Pezzola, A. y Winschel, C. 2004. Estudio espacio-temporal de incendios rurales, utilizando percepción remota y SIG. Estación Experimental Agropecuaria Hilario Ascasubi, Instituto Nacional de Tecnología Agropecuaria. Boletín Técnico № 20. p12.

[16]. Prieto, E., Rivas, B. y Sánchez, J. 2013. Natural polymer grafted with syntethic monomer by microwave for water treatment-a review. Ciencia en Desarrollo, $4(1): 219-240$.

[17]. Rodríguez-Galiano, V., Ghimire, B., Rogan, J., Chica-Olmo, M. y Rigol-Sánchez, J. 2012. An assessment of the effectiveness of a random forest classifier for Land-cover classification. Journal of Photogrammetry and Remote Sensing. 67:93-104.

[18]. Ruíz, V., Savé, R. y Herrera, A. 2013. Análisis multitemporal del cambio de uso del suelo, en el paisaje terrestre protegido Miraflor Moropotente Nicaragua, 19932011. Ecosistemas. 22(3):117-123. 
[19]. Saadat, H., Adamowski, J., Bonnell, R., Sharifi, F., Mohammad, N. y Ale-Ebrahim, S. 2011. Land Use and Land Cover classification over a large area in Iran based on single date analysis of satellite imagery. Journal of Photogrammetry and Remote Sensing. 66(5):608-619.

[20]. Sanmartín-Sierra, D., Angarita-Hernández D. y Mercado-Gómez J. 2016. Estructura y composición florística del bosque seco tropical de Sanguaré-Sucre (Colombia). Ciencia en Desarrollo. 7(2):43-56.
[21]. Sepulveda, O., Suárez, Z., Patarroyo, M., Canaria, L. y Bautista, S. 2015. Estudio del comportamiento e impacto de la climatología sobre el cultivo de la papa y del paso en la región central de Boyacá empleando los sistemas dinámicos. Ciencia en Desarrollo. 6(2):215-224.

[22]. Veraverbeke, S., Gitas, Lo., Katagis, T., Polychronaki, A., Somers, B. y Goossens, R. 2012. Assessing post-fire vegetation recovery using red-near infrared vegetation indices: Accounting for background and vegetation variability. ISPRS Journal of Photogrammetry and Remote Sensing. 68:p. 28-39. 